Rodrigo Bastos Moraes

\title{
Alocação dinâmica de símbolos-piloto para sistemas OFDM de enlace fechado
}

Dissertação apresentada como requisito parcial para obtenção do grau de Mestre pelo Programa de Pós-graduação em Engenharia Elétrica do Departamento de Engenharia Elétrica da PUC-Rio

Orientador: Prof. Raimundo Sampaio Neto 


\title{
Rodrigo Bastos Moraes
}

\section{Alocação dinâmica de símbolos-piloto para sistemas OFDM de enlace fechado}

Dissertação apresentada como requisito parcial para obtenção do grau de Mestre pelo Programa de Pós-graduação em Engenharia Elétrica do Departamento de Engenharia Elétrica do Centro Técnico Científico da PUC-Rio. Aprovada pela Comissão Examinadora abaixo assinada.

\author{
Prof. Raimundo Sampaio Neto \\ Orientador \\ Departamento de Engenharia Elétrica - PUC-Rio \\ Prof. Ernesto Leite Pinto \\ Instituto Militar de Engenharia - IME \\ Prof. José Mauro Pedro Fortes \\ Departamento de Engenharia Elétrica - PUC-Rio \\ Prof. Marcelo Roberto Jimenez \\ Departamento de Engenharia Elétrica - PUC-Rio
}

Prof. José Eugenio Leal

Coordenador Setorial do Centro Técnico Científico - PUC-Rio 
Todos os direitos reservados. É proibida a reprodução total ou parcial do trabalho sem autorização da universidade, do autor e do orientador.

\section{Rodrigo Bastos Moraes}

Graduou-se pela Universidade Federal do Pará (UFPA) em 2005. Em 2006, foi pesquisador visitante no laboratório Broadband Access Research da Ericsson AB, em Estocolmo, Suécia. Ganhou a bolsa FAPERJ Nota 10 e o Travel Grants da Communications Society do IEEE, ambos em 2008.

Ficha Catalográfica

Moraes, Rodrigo B.

Alocação dinâmica de símbolos-piloto para sistemas OFDM de enlace fechado / Rodrigo Bastos Moraes; orientador: Raimundo Sampaio Neto. - Rio de Janeiro : PUC-Rio, Departamento de Engenharia Elétrica, 2009.

v., 93 f: il. ; $29,7 \mathrm{~cm}$

1. Dissertação (mestrado) - Pontifícia Universidade Católica do Rio de Janeiro, Departamento de Engenharia Elétrica.

Inclui referências bibliográficas.

1. Engenharia Elétrica - Tese. 2. Orthogonal Frequency Division Multiplexing;. 3. Estimação de canal;. 4. Sistemas de enlace fechado;. 5. Alocação de pilotos.. I. Sampaio Neto, Raimundo. II. Pontifícia Universidade Católica do Rio de Janeiro. Departamento de Engenharia Elétrica. III. Título. 


\section{Agradecimentos}

Agradeço a minha família, pelo apoio e pela confiança. Sou grato especialmente a minha mãe. Agradeço a minhas tias Cíntia, Lourdes e Nazaré, pelo carinho, companhia e apoio logístico. Nazaré Nobre era uma grande pessoa, e infelizmente recentemente nos deixou.

A Tatiana Pará, pelo companheirismo e amizade. Esse trabalho teria sido muito mais difícil sem a sua companhia.

Sou grato ao professor Raimundo Sampaio, cujas contribuições a esse trabalho foram fundamentais. Fica aqui meu respeito e admiração. Agradeço também a todos os meus professores do CETUC.

Agradeço a FAPERJ, CAPES e CNPQ pelo apoio financeiro.

A meus amigos e professores da UFPA, em especial a turma do projeto DSL, por terem proporcionado um ambiente excelente para o meu desenvolvimento profissional. Agradeço ao professor Aldebaro Klautau, que foi com quem essa história começou.

A meus amigos e colegas do CETUC, principalmente Aline Silva, Davi Guedes, Dick Carrillo, Eleonora Andrade, Mauro Lustosa, Raul Velloso e Tiago Vinhoza. 


\section{Resumo}

Moraes, Rodrigo B.; Sampaio Neto, Raimundo. Alocação dinâmica de símbolos-piloto para sistemas OFDM de enlace fechado. Rio de Janeiro, 2009. 93p. Dissertação de Mestrado - Departamento de Engenharia Elétrica, Pontifícia Universidade Católica do Rio de Janeiro.

Sistemas OFDM têm conseguido atenção dos órgãos internacionais de padronização na última década. Vários trabalhos na literatura tratam sobre como otimizar a transmissão desses sistemas na situação de enlace aberto, ou seja, onde não há comunicação reversa entre transmissor e receptor. Este trabalho foca a utilização de enlace fechado para sistemas de transmissão OFDM, um assunto pouco explorado até agora. Aqui focamos principalmente no método recentemente proposto na literatura de alocação dinâmica de símbolos-pilotos. Foi mostrado recentemente que, para sistemas OFDM coerentes e de enlace fechado, essa opção é a que traz mais ganhos. Os símbolos-piloto são usados para obter amostras do comportamento do canal a fim de detectar os símbolos de dado corretamente. Esses pilotos, porém, não precisam estar dispostos para a melhor estimação possível do canal. Almeja-se apenas uma estimação boa o suficiente. Nesse trabalho propõe-se uma estratégia de alocação de pilotos com o objetivo de minimizar a probabilidade de erro de bits do sistema. Sugere-se também um algoritmo de menor complexidade que mantém grande parte do desempenho da solução ótima. Resultados experimentais mostram ganhos bastante significativos.

\section{Palavras-chave}

Orthogonal Frequency Division Multiplexing; Estimação de canal; Sistemas de enlace fechado; Alocação de pilotos. 


\section{Abstract}

Moraes, Rodrigo B.; Sampaio Neto, Raimundo (Advisor). Dynamic Pilot-Symbol Allocation for Closed-loop OFDM Systems. Rio de Janeiro, 2009. 93p. MSc. Dissertation - Departamento de Engenharia Elétrica, Pontifícia Universidade Católica do Rio de Janeiro.

OFDM systems have gathered quite some attention from international standardization bodies for the past decade. Various works in literature aim at optimizing transmission for these systems in the open-loop scenario, i.e. when there is no feedback communication between transmitter and receiver. This work focuses on the utilization of a closed-loop for OFDM systems, a subject which has not been thoroughly explored so far. Here we primarily focus on the recently proposed method of dynamic pilot-symbol allocation. It was shown in a recent paper that for coherent closed-loop OFDM systems, this option is capable of delivering substantial increases in performance. The pilot-symbols are used to sample channel behavior so that data symbols can be decoded correctly. However, these pilot do not need to be located so as to estimate the channel in the best possible way. The system requires an estimation which is only good enough. In this work, we propose a pilotsymbol allocation strategy with the goal of minimizing the bit error rate. We also suggest a lower complexity algorithm, which attains most of the performance of the optimal solution. Experimental results show significant gains.

\section{Keywords}

Orthogonal Frequency Division Multiplexing; Channel Estimation; Closed-loop systems; Pilot-symbol allocation. 


\section{Sumário}

1 Introdução 12

2 OFDM: Princípios Básicos $\quad 15$

$\begin{array}{ll}2.1 \text { Modelo Contínuo } & 16\end{array}$

2.2 Transmissão paralela vs. Transmissão serial 20

2.3 Modelo Discreto 21

2.4 Modelagem do canal discreto 25

3 Estimação de Canal e Detecção em OFDM com Enlace Aberto 28

3.1 Estimação do canal 29

$\begin{array}{lll}3.2 & \text { Recepção } & 45\end{array}$

3.3 Probabilidade de erro 49

3.4 Experimentos 52

4 Estimação de Canal e Detecção em OFDM com Enlace Fechado 58

4.1 Pré-Equalização 59

4.2 Alocação de Potência 61

4.3 Alocação de pilotos $\quad 62$

$\begin{array}{lll}4.4 & \text { Experimentos } & 74\end{array}$

5 Conclusão $\quad 83$

$\begin{array}{lr}\text { Referências Bibliográficas } & 85\end{array}$

$\begin{array}{ll}\text { A Abreviações } & 88\end{array}$

$\begin{array}{lll}\text { B Operadores Matemáticos } & 89\end{array}$

C Cálculos para o MMSE $\quad \mathbf{9 0}$

$\begin{array}{lll}\text { C.1 MSE } & 90\end{array}$

C.2 Filtro de Wiener $\quad 91$

C.3 MSE para MMSE mais filtro de Wiener 92 


\section{Lista de figuras}

2.1 Modelo de transmissão OFDM contínuo. 17

2.2 Prefixo cíclico para o $n$-ésimo bloco de dados para o modelo contínuo. 17

2.3 À esquerda, ilustração de um sistema com portadora única. O espectro do sinal é seriamente distorcido pelo canal. À direita, sistema de multi-portadoras ortogonais. Sub-canais OFDM encontram canais aproximadamente planos na freqüência. 21

2.4 Modelo de transmissão OFDM discreto. 22

2.5 Prefixo cíclico para o $n$-ésimo bloco de dados para o modelo discreto. 22

2.6 Filtro autoregressivo para a geração do canal Rayleigh. 26

2.7 Autocorrelação para $5 \times 10^{6}$ amostras de uma VA gerada com o modelo autoregressivo com $Q=200$.

3.1 Modelo de comunicações com enlace aberto.

3.2 MSE por tom para $E_{s} / \sigma=10 \mathrm{~dB}$ - 'o' e ' $\times$ ' representam a posição dos pilotos para as alocações não-uniforme e uniforme, respectivamente.

3.3 Diagrama de blocos para a filtragem de Wiener. 40

3.4 Correlação espacial entre sub-canais para $K=64$ e $L=8$. 41

3.5 Diagrama de bloco para recepção em enlace aberto. 45

3.6 Probabilidade de erro para enlace aberto. 52

3.7 MSE para pilotos uniformes e diferentes estimadores. 54

3.8 BER para pilotos uniformes e diferentes estimadores. 54

3.9 BER e MSE para $f_{m}=5 \times 10^{-2}$, estimador ML com filtro de Wiener para três $E_{b} / \sigma$ distintas.

3.10 BER e MSE para $f_{m}=5 \times 10^{-3}$, estimador ML com filtro de Wiener para três $E_{b} / \sigma$ distintas.

3.11 BER e MSE para $f_{m}=5 \times 10^{-6}$, estimador ML com filtro de Wiener para três $E_{b} / \sigma$ distintas.

4.1 Enlace fechado.

4.2 Diagrama de bloco para a pré-equalização. 60

4.3 Diagrama de blocos para a alocação dinâmica de potência. 62

4.4 Diagrama de blocos para a alocação dinâmica de pilotos. 64

4.5 Degrau no tempo com dois instantes tem apenas um nulo espectral. 68

4.6 Degrau no tempo com $L$ instantes tem $L-1$ nulos espectrais. $\quad 68$

4.7 SNR vs. probabilidade de erro. $\quad 70$

4.8 Probabilidade de erro para enlace fechado. $\quad 71$

4.9 Exemplo do funcionamento do BITE.

4.10 Erro médio quadrático de estimação do canal: comparação entre os diferentes esquemas de uso do enlace reverso. Estimador ML simples. 76

4.11 Probabilidade de erro de bit: comparação entre os diferentes esquemas de uso do enlace reverso. Estimador ML simples.

4.12 Erro médio quadrático: comparação entre as situações de estimador ML simples e ML mais filtro de Wiener. 
4.13 Probabilidade de erro de bit: comparação entre as situações de estimador ML simples e ML mais filtro de Wiener.

4.14 Histograma dos módulos dos canais que carregam dados para (A) enlace aberto, (B) Panah, ML e Wiener e (C) Proposto, ML e Wiener. 80

4.15 Visão aproximada do histograma. $\quad 80$

4.16 Grid para o enlace aberto. $\quad 81$

4.17 Grid para o Panah, ML. $\quad 81$

4.18 Grid para o Panah, ML e Wiener. $\quad 81$

4.19 Grid para o Proposto, ML e Wiener. 81

4.20 Erro médio quadrático: $K=64$ e $K_{p}=16$. 82

4.21 Probabilidade de erro de bit: $K=64$ e $K_{p}=16$. 


\section{Lista de tabelas}

3.1 Ruído total por tom para os diferentes estimadores 50

3.2 Parâmetros para a simulação 53

4.1 Parâmetros para a simulação: comparação entre os esquemas de uso do enlace reverso 
Deus ao mar o perigo e o abismo deu, Mas nele é que espelhou o céu.

Fernando Pessoa, 1888-1935. 